In a similar manner the non-existence of the compound $\mathrm{LiNH}_{3}$ is established. In this case independent evidence is given which shows conclusively that in a system containing lithium and a small molecular per cent. of ammonia a saturated solution of the metal in ammonia is formed. This result is in agreement with the phase relationships existing in the system and demonstrates the inapplicability of the method employed by Moissan in obtaining and identifying the supposed compound of lithium and ammonia.

It is shown that calcium forms a solid compound with ammonia whose composition is represented by the formula $\mathrm{Ca}\left(\mathrm{NH}_{3}\right)_{6}$. "lhe optical properties of this compound are apparently identical with those of its satura ted solution in ammonia, and like its solution, the compound exhibits metallic conduction.

The vapor pressures of saturated solutions of lithium and of $\mathrm{Ca}\left(\mathrm{NH}_{3}\right)_{6}$ in ammonia have been determined, as well as the dissociation pressures of the compound itself. The heats of formation of the corresponding solutions and of the compound from metal and gascous ammonia are calculated to be 8700 , 10230, and 5460 calories per gram-molecule of an1monia, respectively.

The constitution of the compound $\mathrm{Ca}\left(\mathrm{NH}_{3}\right)_{0}$ is discussed. It appears that this compound is of the nature of a solvate, corresponding, perhaps, to an ammoniated calcitim ion. It is suggested that the compound be called calcium hexammoniate in order to take account of these relations in the nomenclature.

Boston, February 6th, 1908.

[Contributions from the Research Laboratory Ol Physical Chemistry of the Massachusetts INstitute of Technology. No. 25.]

\title{
THE OSMOTIC PRESSURE OF CONCENTRATED SOLUTIONS, AND THE LAWS OF THE PERFECT SOLUTION.
}

\author{
By GILbert Newton I,Ewrs. \\ Received March 5, 1908.
}

The laws of the infinitely dilute solution have been thoroughly established. 'There can be no reasonable doubt as to the accuracy of Henry's law for the vapor pressure of the solute, Raoult's law for the vapor pressure of the solvent, or van't Hoff's law for the osmotic pressure, in the case of an infinitely dilute solution. In fact if any one of these laws is shown to be correct, the other two must follow as a direct consequence of the laws of thermodynamics.

Unfortunately, we never work with an infinitely dilute solution, and too little attention has been given to the question of the validity or even the mutual compatibility of the laws just mentioned in concentrated solutions and even in the so-called dilute solutions. 
There is a well known thermodynamic relation between the osmotic pressure of a solution and the lowering of the vapor pressure of the solvent, which enables us, in every case, regardless of the concentration of the solution, to calculate the osmotic pressure when the vapor pressure lowering is known, and vice iersa. It is therefore possible to calculate the osmotic pressure of a given solution, first, on the assumption that van't Hoff's law $^{1}$ is correct, and second, on the assumption that Raoult's law is correct. It is commonly supposed that for fairly dilute solutions these two methods of calculating the osmotic pressure give identical results, but this is not the case. For example, the osmotic pressures calculated in these two ways for a normal solution of cane sugar differ by 20 per cent. and even at the dilution of 0.005 normal the difference is still o.I per cent. It is obvious, therefore, that even in that region to which we are accustomed to apply the term, "dilute solution," the law of Raoult and the law of van't Hoff are not compatible. If one is true, the other must be false. What then shall we regard as an ideal or perfect solution, one that obeys the law of Raoult or one that obeys the law of van't Hoff, or shall we choose another criterion which differs from both of these?

Morse and Frazer, ${ }^{3}$ who have recently succeeded in measuring osmotic pressures up to 25 atmospheres by a direct method, propose to replace the law of van't Hoff by the following equation, which gives values for the osmotic pressure more in accord with those obtained experimentally in the case of sugar and glucose:

$$
\Pi=\frac{n \mathrm{RT}}{\mathrm{V}^{\prime}}
$$

Here $\mathrm{V}^{\prime}$ is not the volume of solution but the volume of pure solvent in which $n$ mols of solute are dissolved. These authors propose, therefore, to substitute for the system in which concentrations are expressed in mols of solute in one liter of solution (volume normal system) another in which concentrations are expressed in mols of solute dissolved in one liter of pure solvent (weight normal system). In most cases the difference between these two systems is much less than it is in the case of the two substances of high molecular weight investigated by Morse and Frazer. Thus a weight normal solution of sugar is only 0.82 volume normal, a difference of about 20 per cent., but in the case of methyl alcohol, ammonia and hydrochloric acid, substances of small molecular weight se-

${ }^{1} \Pi=n \mathrm{RT} / \mathrm{V}$, where $\mathrm{II}$ is the osmotic pressure, $\mathrm{R}$ the gas constant, $\mathrm{T}$ the absolute temperature, and $n$ is the number of mols of solute dissolved in the volume $V$ of the solution.

$2\left(p_{0}-p\right) / p_{0}=n_{1} /\left(n+n_{1}\right)$, where $p_{0}$ is the vapor pressure of the solvent in the pure ${ }_{2}$ state, $p$ that of the solvent from the solution, and $n_{1}$ is the number of mols of solute dissolved in $n$ mols of solvent.

'Amer. Chem. J., 34, I (1905); 37, 324, 425, 558; 38, I7.5 (1907). 
lected at random for this calculation, the difference in concentration of weight normal and volume normal amounts to only 2,4 , and 2 per cent., respectively. It is fortunate, however, that they did study those very substances in which the difference between the two systems is most pronounced, for we are thus forced to face certain questions concerning moderately dilute solutions which have been too often evaded.

It will be the purpose of this paper not only to find what theoretical justification there may be for the above modification of the van't Hoff equation, but also to determine in general which of the various laws of solutions may be most suitably chosen to define the perfect solution.

Before beginning this inquiry it may be well to discuss bricfly another question raised by Morse and Frazer, who write with some disparagement of the methods of determining osmotic pressure which rest upon thermodynamic calculations. Without undervaluing in any degree the importance of direct measurements of a quantity which has played so important a part in the development of modern chemistry as osmotic pressure, it must nevertheless be clefinitely affirmed that we have at our disposal several means of determining the osmotic pressure which are readily capable of furnishing results many times as accurate as any yet obtained by direct measurement. These methods will, therefore, be briefly considered in the following section:

\section{Direct and Indirect Osmotic Pressure Measurements.}

The exact definition of osmotic pressure, and some of the thermo dynamic relations in which the osmotic pressure is involved will be discussed briefly in notes at the enc of this paper. There it will be shown that the osmotic pressure of an aqueous solution may be obtained at once from the freezing point by means of the equation

$$
\Pi=\mathrm{I} 2.06 \Delta-0.02 \mathrm{I} \Delta^{2},
$$

where $\Pi$ is the osmotic pressure in atmospheres and $\Delta$ is the lowering of the freezing-point in centigrade degrees. ${ }^{1}$ From this equation the osmotic pressure of any solution up to ten or fifteen times normal may be obtained with an accuracy which depends only upon the precision of the freezing-point determinations and upon the accuracy of the value used for the heat of fusion of ice. Since the error in the latter quantity

1 If we assume that at infinite dilution van't Hoff's law holds exactly, and take $\mathrm{R}=0.08207$ liter atmospheres per degree, from the work of D. Berthelot (Z. Elektrochem., I0, 621, 1904), then we find from equation ( $\mathrm{I}$ ) that the molecular lowering of the freezing-point of any aqueous solution at infinite dilution is $1.85^{\circ}$, which differs materially from the value commonly used, namely, i.85. The latter value is used by Morse and Frazer, but they should use the value 1.843 , for they do not employ the international atomic weights but those based on hydrogen as unity. The mol is therefore reckoned in the latter system, and not in the customary one, in Tables I and II where Morse and Frazer's data are used. 
is probably not more than about o.I per cent., it is obvious that except for the most dilute solutions osmotic pressures may be found in this way with an accuracy which is more than ten times as great as Morse and Frazer claim for their direct measurements. It is interesting to compare the osmotic pressures obtained by Morse and Frazer with those calculated by equation (I) from the freezing-point measurement of the same authors. This comparison is made in Tables I and II. The first TABlE I.-CANE Sugar.

$\begin{array}{cc}\text { M. } & \Delta . \\ 0.1 & 0.195 \\ 0.2 & 0.392 \\ 0.3 & 0.585 \\ 0.4 & 0.784 \\ 0.5 & 0.985 \\ 0.6 & 1.19 \\ 0.7 & 1.39 \\ 0.8 & 1.62 \\ 0.9 & 1.83 \\ 1.0 & 2.07 \\ & \\ 0.867 & 1.77 \\ 1.25 & 2.68 \\ 1.54 & 3.42 \\ 1.63 & 3.63 \\ 2.10 & 4.88\end{array}$

\begin{tabular}{|c|c|}
\hline ПI obs. & $\Pi$ Calc. \\
\hline 2.44 & $2 \cdot 35$ \\
\hline 4.80 & $4 \cdot 73$ \\
\hline $7 \cdot 16$ & 7.05 \\
\hline 9.40 & 9.45 \\
\hline 11.8 & II. 8 \\
\hline 14.2 & 14.3 \\
\hline I 6.8 & I 6.7 \\
\hline 19.3 & r9.5 \\
\hline 22.1 & 22.0 \\
\hline 24.8 & 24.9 \\
\hline$\cdots \cdots$ & $21 \cdot 3$ \\
\hline$\ldots$ & 32.2 \\
\hline$\ldots$ & 41.0 \\
\hline & $43 \cdot 5$ \\
\hline$\cdots \cdots$ & $5^{8} .4$ \\
\hline
\end{tabular}

TABLE II.-GLUCOSE.

M. $\Delta$

$\begin{array}{ll}0.1 & 0.192 \\ 0.2 & 0.386 \\ 0.3 & 0.576 \\ 0.4 & 0.762 \\ 0.5 & 0.952 \\ 0.6 & 1.15 \\ 0.7 & 1.34 \\ 0.8 & 1.53 \\ 0.9 & 1.72 \\ 1.0 & 1.92\end{array}$

II Obs.

II Calc.

2.32

4.65

4.65

7.01

$9 \cdot 30$

II . 6

14.0

I6. 4

I 8.8

6.94

9.18

II. 5

I3.8

I6. I

21.2

18.4

23.6

20.7

23. 1

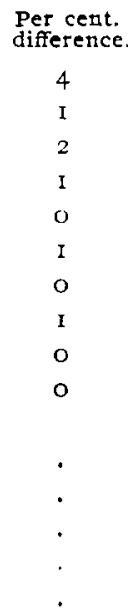

column gives $\mathrm{M}$, the number of mols of solute in one liter of water, the second the lowering of the freezing-point, the third the osmotic pressure directly measured, the fotirth that calculated from $\Delta$, and the fifth gives in round numbers the percentage difference between the observed and calculated values. In Table I, I have also given (below the line) the osmotic pressure of cane sugar solutions calculated from the freezingpoint measurements of Ewan. ${ }^{1}$ These seem in perfect accord with the values of Morse and Frazer and extend to higher concentrations. It

${ }^{1} Z$. physik. Chem., 3I, 22 (1899). 
is to be noted that each calculated value is obtained for the temperature at which the solution in question freezes, while the observed values were found at a few tenths of a degree above zero, but the correction for this small temperature difference is too small in comparison with the experimental errors to be considered.

It is apparent that the observed and calculated values for the osmotic pressure agree within the limits of crror of the former. 'The tables indicate, moreover, that the experiments with glucose were somewhat less reliable than those with cane sugar.

Since, therefore, freezing-point measurements offer a simple and exact means of determining the osmotic pressure at the freezing-point, it is possible from them to determine the osmotic pressure at other temperatures, if we know its temperature coefficient. Morse and Frazer have considered it impossible to predict the value of this coefficient, but they have overlooked the simple thermodynamic equation, which may be derived immediately from the familiar energy equation of Helmholtz, namely,

$$
\mathrm{II}-q=\mathrm{T} \frac{d \mathrm{II}}{d \mathrm{~T}^{\prime}}
$$

where $\Pi$ is the osmotic pressure, ' $T$ ' the absolute temperature and $q$ is the heat of dilution, that is, the heat evolved when one cc. of solvent is added to a large quantity of the solution. This quantity $q$ is known for a large number of solutions and in any case may be very easily determined. For cane sugar we have very accurate knowledge of this quantity for one temperature, $15^{\circ}$, from the independent but entirely accordant work of von Stackelberg ${ }^{1}$ and Ewan. ${ }^{2}$ According to their measurements in the case of a weight normal solution $q$ is equal to 0.12 cal. or $5 \mathrm{cc}$-atmos. Substituting the latter value in equation (2) and calling II at $\mathrm{I} 5^{\circ}$ approximately 24 atmos., which according to the experiments of Morse and Frazer cannot be far wrong, we find

$$
\frac{d \mathrm{II}}{d \mathrm{~T}}=\begin{gathered}
24-5 \\
273+\mathrm{I} 5
\end{gathered},
$$

or about 0.07 atmos. per degree. In other words, while the osmotic pressure of an ideal solution at $15^{\circ}$ changes 0.35 per cent. per degree the normal sugar solution changes only 0.27 per cent. per degree. Unfortunately we do not know the heat of dilution of sugar solutions at lower temperatures, but since in other cases von Stackelberg has shown that it increases with decreasing temperature, it is probable that it does in this case also. The temperature coefficient of osmotic pressure will therefore probably become smaller at lower temperatures and may even become negative (when $q>\mathrm{II}$ ), which would explain the surprising fact

1 Z. physik. Chem., 26, 533 (1898).

${ }^{2}$ Loc. cit. 
discovered by Morse and Frazer that the osmotic pressure of cane sugar is about the same at $0^{\circ}$ as it is at $25^{\circ}$.

Since the heat of dilution may be very readily measured at any temperature, we have by its means a remarkably simple method of determining the osmotic pressure at any temperature, if it is known at one.

For obtaining the osmotic pressure of a solution at any temperature there is another perfectly general indirect method which has been frequently employed ${ }^{1}$ and recently has been improved to such a point that it rivals in accuracy the freezing-point method. ${ }^{2}$ It depends upon the thermodynamic relation between the vapor pressure from a solution and the osmotic pressure, which may be expresssed in the equation ${ }^{3}$

$$
\Pi-\frac{\mathrm{I}}{2} \alpha \Pi^{2}=\frac{\mathrm{RT}}{\mathrm{V}_{0}} \ln \frac{p_{0}}{p} .
$$

Here $\Pi$ is again the osmotic pressure, $\alpha$ is the coefficient of compressibility of the solvent, $\mathrm{V}_{\circ}$ is its molecular volume, $\ln$ stands for natural logarithm and $p_{0}$ and $p$ are respectively the vapor pressure of the solvent in the pure state and in the solution. Several applications of this equation will be made in the following section.

\section{The Law of Ideal Solutions.}

What we shall call a perfect or ideal solution is somewhat a matter of choice. We might define as an ideal solution one which obeys the law of van't Hoff, or the modified form of this law proposed by Morse and Frazer, or the law of Raoult, or the law of Henry. These laws are essentially identical for the infinitely dilute solution, but for a solution of finite concentration we are at liberty to choose one but not all of these laws to define the ideal solution. No one of them is true for every solution at every concentration, and we must therefore choose that one which holds most nearly for the greatest number of substances over the widest limits of concentration.

I shall attempt to show that the most fundamental law of solutions and the one by which the perfect solution is best defined is the following modification of the law of Raoult. At constant pressure and tempera-

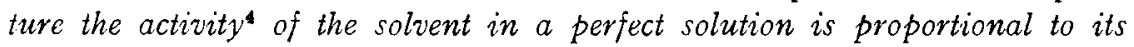
mol fraction. That is,

$$
\xi=\xi_{\circ} N \text {, }
$$

where $\xi$ is the activity of the solvent in the solution, $\xi_{0}$ the activity of the pure solvent, and $\mathrm{N}$, the mol fraction, is the number of mols of sol-

'See for example, Noyes and Abbott, $Z$. physik. Chem., 23, 56 ( 1897 ).

${ }^{2}$ See Smits, $Z$. physik. Chem., 5r, 33 (1905).

' For the development of this equation, see note 3 , at the end of this paper.

- For a definition of the term activity see Lewis, "Outlines of a New System of Thermodynamic Chemistry," Proc. Amer. Acad., 43, 259 (1907); and Z. physik. Chem., 6r, 129 (1907); C. A., I908, 611. 
vent in one nol altogether of solvent and solute. Since, however, the conception of activity is new, and since if the vapor of the solvent obeys the gas law the activity is proportional to the vapor pressure, we may with sufficient exactness for our present purposes, substitute the vapor pressure of the solvent for its activity and write

$$
p=p_{0} N
$$

that is, in a perfect solution the vapor pressure of the solvent is proportional to its mol fraction. " Thus in a solution containing o.I mol solute to $0.9 \mathrm{~mol}$ solvent, $N=0.9$ and the vapor pressure of the solvent should be nine-tenths of its vapor pressure in the pure state, or if the solution contains $0.25 \mathrm{~mol}$ solute to $0.75 \mathrm{~mol}$ solvent $p$ should be $0.75 p_{0}$. This is simply a statement of Raoult's law in its simplest form. ${ }^{2}$

There are no cases in which the law of van't Hoff or the modified form of this law proposed by Morse and Frazer have been shown to hold at concentrations higher than normal. (In a normal solution in water the mol fraction of the solute is about 0.02 .)

Indeed, at very high concentrations van't Hoff's law cannot hold, for the osnotic pressure of a solution approaches infinity as the percentage of solvent approaches zero, while the osmotic pressure calculated from the van't Hoff equation never exceeds a few hundred atmospheres even when we approach the condition of pure solute. On the other hand, it will be shown presently that the law proposed by Morse and Frazer ordinarily gives, at higher concentrations, osmotic pressures far higher than those which actually exist. But often the law of Raoult (and the modified law of Henry) has been shown to hold at all concentrations from o per cent. to roo per cent. of solute, and while in many other cases this

1 The point of view here adopted is practically identical with that which for several yeurs has been advocated by J. J. van Laar in numerous publications.

${ }^{2}$ It is important to note that equation (4) leads us immediately to a simple equation for the activity or the vapor pressure of the solute. In the paper previously referred to I have proved the following exact equation for the change in the activity of each component of a binary mixture with change of composition, namely,

$$
\mathrm{N}_{1} d \ln \xi_{1}+N d l n \xi=0 \text {, }
$$

where $N_{1}$ is the mol fraction and $\xi_{1}$ the activity of one constituent which we will call the solute, and $\mathrm{N}$ and $\xi$ are the corresponding terms for the other constituent which we will call the solvent. Now, when equation (4) is true, $d l n \xi=d l n N$. Substituting in the above equation and noting that by definition $N_{1}=1-N$ we find

$$
\begin{gathered}
\mathrm{N}_{1} d l n \xi_{1}+d \mathrm{~N}=0, \text { or } \\
d l n \xi_{1}=d l n \mathrm{~N}_{1}, \text { or } \\
\xi_{1}=\mathrm{KN},
\end{gathered}
$$

where $\mathrm{K}$ is a constant. We see therefore that in a perfect solution it is also true that the activity of the solute is proportional to its mol fraction. If we substitute $p_{1}$, the vapor pressure of the solute, for $\xi_{1}, p_{1}=\mathrm{KN}_{1}$, which is, in a slightly modified form, the law of Henry. In other words, if both vapors obey the gas law, the law of Henry may be derived thermodynamically from the law of Raoult and must hold if that law does. 
law does not hold, the greatest deviations are always found in those cases in which we have reason to believe that the solvent and the solute form complex compounds either with themselves or with each other.

Many illustrations might be given to show the remarkable scope of Raoult's law. I will choose a binary mixture which has been studied more carefully over a wide range of concentration than any other, namely, benzene and ethylene chloride. The vapor pressures are taken from the excellent paper of Zawidski. ${ }^{2}$ We will call benzene the solvent and ethylene chloride the solute. In Table III, in which the data marked by Zawidski as questionable are omitted, the first column gives the number of grams of solute to one gram of solvent, the second gives the partial vapor pressure of the solvent at $50^{\circ}$, and the third gives the molecular weight of ethylene chloride calculated from the vapor pressures by Raoult's law. The calculated molecular weights are constant, even up to the highest concentration, where the solute constitutes over 90 per cent. of the solution. The average of these calculated molecular weights is 99. I while the actual molecular weight of ethylene chloride is 99.0. (We have therefore every ground for believing that also in the pure state ethylene chloride exists in the form of simple molecules.)

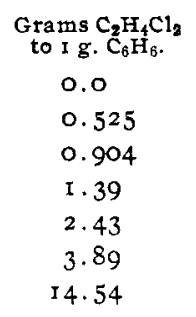

TABLE III.

$\begin{array}{cc}\text { p. of } \mathrm{C}_{6} \mathrm{H}_{6} . & \mathrm{M}_{2} . \mathrm{W} \\ 268.0 & \ldots \ldots \\ 189.8 & 99.4 \\ 156.0 & 98.2 \\ 127.8 & 98.7 \\ 92.4 & 99.3 \\ 65.9 & 99.0 \\ 21.8 & 100.0\end{array}$

Average, 99.1

Theoretical, 99.0

If then we define a perfect solution as one which obeys Raoult's law, ${ }^{2}$ it is interesting to find what the law is connecting osmotic pressure and concentration in a perfect solution. This law, which is less simple than either the'law of van't Hoff or that of Morse and Frazer, may be derived directly from equations (3) and (5), and is

or

$$
\Pi-\frac{1}{2} \alpha \Pi^{2}=-\frac{\mathrm{RT}}{\mathrm{V}_{0}} \ln \mathrm{N}
$$

2. physik. Chem., 35, 129 (1900).

2 Strictly speaking, we define a perfect solution as one which obeys equation (4) rather than equation (5), but the more precise method which employs the activity instead of the vapor pressure leads to exactly the same equation for the osmostic pressure as we shall derive here. 


$$
\mathrm{II}-{ }_{2}^{\mathrm{I}} \alpha \mathrm{II}^{2}=-\frac{\mathrm{RT}}{\mathrm{V}_{\mathrm{o}}} \ln (\mathrm{I}-\mathrm{N}),
$$

where $V_{\circ}$ is the molecular volume and $\alpha$ the compressibility of the solvent.

In lable IV the osmotic pressures of cane sugar solutions are calculated from equation (7). The first column gives the weight normal concentration; the second, the volume normal; the third, $\mathrm{N}_{1}$, the mol fraction of solute; the fourth, the osmotic pressures calculated by the van't Hoff equation; the fifth, those calculated by the equation of Morse and Frazer; the sixth, those calculated by equation (7); the seventh, Morse and Frazer's observed values.

$$
\text { TABLE IV. }
$$

$\begin{array}{ccccccc}\begin{array}{c}\text { Conc. } \\ \text { wt. norm. }\end{array} & \begin{array}{c}\text { Conc. } \\ \text { vol. norm. }\end{array} & \begin{array}{c}\text { Mol fraction } \\ \text { of solute. }\end{array} \text { II } & \text { II } & \text { II } & \text { II } \\ 0.1 & 0.098 & 0.00180 & 2.34 & 2.41 & 2.4 I & 2.40 \\ 0.2 & 0.192 & 0.00358 & 4.58 & 4.81 & 4.80 & 4.74 \\ 0.3 & 0.282 & 0.00537 & 6.73 & 7.23 & 7.21 & 7.23 \\ 0.4 & 0.369 & 0.00715 & 8.81 & 9.64 & 9.60 & 9.67 \\ 0.5 & 0.452 & 0.00892 & 10.8 & 12.0 & 12.0 & 12.1 \\ 0.6 & 0.532 & 0.0107 & 12.7 & 14.5 & 14.4 & 14.4 \\ 0.7 & 0.610 & 0.0124 & 14.5 & 16.8 & 16.7 & 16.9 \\ 0.8 & 0.684 & 0.0142 & 16.3 & 19.3 & 19.2 & 19.4 \\ 0.9 & 0.756 & 0.0159 & 18.1 & 21.7 & 21.5 & 21.8 \\ 1.0 & 0.825 & 0.0177 & 19.7 & 24.1 & 23.9 & 24.5\end{array}$

While the values given by the equation of van't Hoff differ from those observed by nearly 25 per cent. at the higher concentrations, it will be seen that the pressures given in the fifth and sixth columns agree throughout with the observed values, within the limits of experimental error, and differ from each other by only one per cent. even at normal concentration.

This agreement between the osmotic pressures calculated from the equation of Morse and Frazer and those calculated by equation ( 7 ) will always be found at moderate concentrations, as the following consicierations show. The second term in equation (7), except at the very nighest concentrations, is comparatively insignificant, amounting uswally to only a few per cent. of the value of $\Pi$ even when the osmotic pressure is as high as a thousand atmospheres. At moderate concentrations we may, therefore, neglect this term and write equation ( 7 ) in the form,

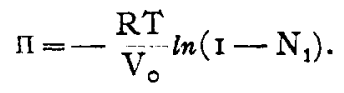

Now the equation of Morse and Frazer may be written in the form

$$
\Pi=\frac{R^{\top} T}{\bar{V}_{0}}\left(\frac{N_{1}}{I-N_{1}}\right) \text {, }
$$


for $\frac{N_{1}}{I-N_{1}}$ is the number of mols of solute to one mol of solvent and $V_{0}$ is the volume of one mol of pure solvent.

Equation (8) developed in series gives

$$
\mathrm{n}=\frac{\mathrm{RT}}{\mathrm{V}_{0}}\left(\mathrm{~N}_{1}+\frac{\mathrm{I}}{2} \mathrm{~N}_{1}^{2}+\frac{\mathrm{I}}{3} \mathrm{~N}_{1}^{3}+\ldots\right) \text {, }
$$

and similarly equation (9) gives,

$$
\Pi=\frac{\mathrm{RT}^{\top}}{\mathrm{V}_{\mathrm{o}}}\left(\mathrm{N}_{1}+\mathrm{N}_{1}{ }^{2}+\mathrm{N}_{1}{ }^{3}+\ldots\right) .
$$

These equations differ only in the higher powers of $N_{1}$ and thereforc give identical results at such concentrations that the terms containing these higher powers are negligible. When the mol fraction of the solute is 0.02 the values of $\pi$ calculated from these equations differ by one per cent. For all more dilute solutions, therefore, the osmotic pressure of a perfect solution may be calculated within one per cent. from the equation of Morse and Frazer.

At higher concentrations, however, the difference between these two equations becomes very great, as is shown in Tables V and VI. Table $\mathrm{V}$ deals with solutions of ethylene chloride in benzene, and simply restates in a new way the facts brought out in Table III. Table VI contains data on solutions of propylene bromide in ethylene bromide. In both tables the first column gives the mol fraction of solute; the second, the partial vapor pressure of the solvent, taken from the work of Zawidski; ${ }^{1}$ the third, the osmotic pressure calculated by the van't Hoff equation; the fourth, that calculated by the equation of Morse and Frazer; the fifth, that calculated by equation (7), while the last column gives the actual osmotic pressure obtained thermodynamically from the vapor pressures by means of equation (3). The molecular volumes of benzene and ethylene chloride at $50^{\circ}$ are taken, respectively, as 0.092 and 0.082 liter, and the coefficient of compressibility of benzene as $0.000 \mathrm{I}$. The molecular volumes of ethylene and propylene bromides at $85^{\circ}$ are taken, respectively, as 0.092 and $0.1{ }_{3} 3$ liter, and the coefficient of compressibility of ethylene bromide as $0.000,06$.

\section{TABLE V.}

\begin{tabular}{|c|c|c|c|c|c|}
\hline $\mathrm{N}_{1}$. & & $\prod_{\text {Van't Hoff. }}$ & $\begin{array}{l}\text { II. } \\
\text { Morse and Frazer. }\end{array}$ & $\underset{\text { Lewis. }}{\Pi \text {. }}$ & $\underset{\text { Found }}{\Pi \text {. }}$ \\
\hline 0.0 & 268.0 & .. & & .. & \\
\hline 0.293 & 189.8 & $9 \mathrm{I}$ & 120 & IOI & 100 \\
\hline 0.416 & 156.0 & 128 & 205 & 157 & 158 \\
\hline 0.522 & 127.8 & 160 & 315 & 215 & 217 \\
\hline 0.657 & 92.4 & 196 & 549 & $3 \times 3$ & 310 \\
\hline 0.754 & 65.9 & 223 & 880 & 413 & 406 \\
\hline .920 & 21.8 & 268 & 3290 & 743 & 735 \\
\hline
\end{tabular}
$\mathrm{C}_{2} \mathrm{H}_{4} \mathrm{Cl}_{2}$ in $\mathrm{C}_{6} \mathrm{H}_{6}$ at $50^{\circ}$.

1 Omitting the values which the author marks as questionable. 


\begin{tabular}{|c|c|c|c|c|c|}
\hline & & $\begin{array}{r}\text { TABLE } \\
\mathrm{H}_{6} \mathrm{Br}_{2} \text { in } \mathrm{C}_{2} \mathrm{~F}\end{array}$ & $\begin{array}{l}\text { VI. } \\
\mathrm{I}_{4} \mathrm{Br}_{2} \text { at } 85^{\circ} \text {. }\end{array}$ & & \\
\hline $\mathrm{N}_{1}$ & $p_{2}$. & $\begin{array}{l}\text { II. } \\
\text { van't Hoff. }\end{array}$ & $\begin{array}{l}\Pi \text { Morse and Frazer. } \\
\text { Men }\end{array}$ & $\begin{array}{l}\text { Il. } \\
\text { Lewis. }\end{array}$ & $\begin{array}{l}\text { II. } \\
\text { Found. }\end{array}$ \\
\hline 0.0 & 172.6 & . & $\ldots$ & . & . \\
\hline 0.147 & I 45.1 & 47 & 55 & $5 \mathrm{I}$ & 55 \\
\hline 0.222 & 132.2 & 69 & $9 \mathrm{I}$ & 80 & 86 \\
\hline 0.298 & I 2 I , I & 90 & $13^{6}$ & I 13 & I 14 \\
\hline 0.412 & IOI I & I 2 I & 224 & 171 & I 7.3 \\
\hline 0.526 & 81.9 & 150 & $35 \mathrm{I}$ & 241 & 240 \\
\hline 0.620 & 64.0 & I 73 & .520 & 313 & 359 \\
\hline 0.720 & 48.0 & 198 & 820 & $4 \mathrm{I} 2$ & 414 \\
\hline 0.800 & $34 \cdot 3$ & 218 & 1280 & 522 & 52.5 \\
\hline 0.860 & $23 \cdot 5$ & 232 & I 960 & 640 & 649 \\
\hline 0.915 & I 3.8 & $24 \mathrm{I}$ & 3440 & 806 & 827 \\
\hline
\end{tabular}

We see from these tables how closely in these two cases the actual osmotic pressures agree with those calculated by equation ( 7 ), and how far from the truth are the pressures calculated both by the van't Hoff equation and by that of Morse and Frazer. These two solutions are, according to our definition, perfect solutions, within the limits of experimental error, for all concentrations from o to over 90 per cent. of solute. Since, moreover, these cases are not unique but have been chosen out of a large number of similar cases merely because of the greater experimental care with which they have been investigated, it is to be presumed that even those solutions which are not perfect at all concentration will, on the average, follow the law expressed in equation (7) to higher concentrations than they will the law of van't Hoff or that of Morse and Frazer.

In view of the experiments of Morse and Frazer, it has recently been proposed $^{1}$ that in the ordinary equations of chemical equilibrium the concentrations expressed in the volume normal system should be replaced by those expressed in the weight normal system. This is undoubtedly an improvement, but the equations thus obtained arc not entirely cor rect, cven when all the substances concerned are present as perfect solutions.

In order to find an exact equation, let us consider a reaction occurring as follows:

$$
x_{1} \mathrm{X}_{1}+x_{2} \mathrm{X}_{2}+=x_{3} \mathrm{X}_{3}+x_{4} \mathrm{X}_{4}+\quad,
$$

where $x_{1}$ mols of $\mathrm{X}_{1}$ combine with $x_{2}$ mols of $\mathrm{X}_{2}$ to form $x_{3}$ mols of $\mathrm{X}_{3 ;}$, etc. It is readily seen from the considerations advanced in this paper and from the thermodynanic laws of chemical equilibrium, ${ }^{2}$ that the general equation of chemical equilibrium, regardless of the concentra-

${ }^{1}$ Walden: 2 . physik. Chem. 58, 50o. This paper also contains a letter from van't Hoff on this subject.

${ }^{2}$ Lewis: Loc. cit., equation (XXIII). 
tions of the reacting substances, provided that they are all present as perfect solutions, is as follows:

$$
\begin{aligned}
& \mathrm{N}_{3}^{x_{3}} \mathrm{~N}_{4}^{x_{4}} \cdots \\
& \mathrm{N}_{1}^{x_{1}} \mathrm{~N}_{2}^{x_{2}} \ldots
\end{aligned}=\mathrm{K} \text { (a constant), }
$$

where $N_{1}, N_{2}$, etc, are the respective mol fractions of $X_{1}, X_{2}$, etc.

'This, then, is the form which the mass law assumes when the substances concerned form perfect but not infinitely dilute solutions, and for suli cases it is rigorously exact.

\section{Note $r$.}

If a solution and the pure solvent are separated by a semiperinable membrane the solvent will flow through the membratle into the solution, where its escaping tendency is less. The only way of preventing this How is to nuake the escaping tendency of the solvent the same on both sides of the nembrane. There are two simple ways of accomplishing this, (I) to increase the pressure on the solution until the escaping ten dency of the solvent in the solution is raised to equal that of the solvent in the pure state, (2) to diminish the pressure on the pure solvent until its escaping tendency is lowered to equal that of the solvent in the solution.

The osmotic pressure may therefore be defined in two ways, (1) as usually defined it is the increase in the pressure on the solution neces sary to bring the latter into equilibrium with the solvent; (2) Noyes, however, prefers to define the osmotic pressure as the diminution in the pressure on the solvent necessary to bring it into equilibrium with the solution. Neither of these definitions is entirely free from objections, but since the second one permits a much simpler mathenatical treat. ment than the first, it has been adopted throughout this paper. The osmotic pressures defined in these two ways differ only when there is at total change of volume when a small quantity of solvent is adced to a solution. There is no such volume change in the case of sugar and ghin cose as shown by the experiments of Morse and Frazer and of Ewan. We have been justified, therefore, in applying our equations, which in. volve the osmotic pressure according to the second definition, to the results of Morse and Frazer, who worked with the osmotic pressure of the first definition.

\section{Note 2.}

The exact equation connecting osmotic pressure and freezing point may be found as follows: Let us consider an aqueous solution in equilibrium with ice at the temperature ' $T$ and the pressure $p$, and a lso in equilib. rium with these, through a semipermeable membrane, pure water al. the same temperature and at the pressure p 4 , If obliously being the osmotic pressure. Now if the temperature changes by all and the pres

${ }^{1}$ Z. physik. Chem., 35, 707 (1900) 
sure on the solution and ice remains equal to $P$, that on the water must be changed in order to maintain equilibrium. The necessary change in pressure we will call $d \Pi$. Since the water and ice are in equilibrium at the beginning, the activity $\xi$ of the water and the activity $\xi^{\prime}$ of the ice must be equal, and these, moreover, must remain constant as the temperature changes. Hence,

and

$$
\xi=\xi^{\prime}
$$

$$
d \xi=d \xi^{\prime}
$$

or,

$$
d \ln \xi=d \ln \xi^{\prime} .
$$

Now the change in the activity of the ice is due only to the change of temperature, that is,

$$
d \ln \xi^{\prime}=\left(\frac{\partial \ln \xi^{\prime}}{\partial \mathrm{T}}\right) d \mathrm{~T},
$$

but the activity of the water is changed both by the change in temperature and the change in pressure, that is,

$$
d \ln \underline{\xi}=\left(\frac{\partial \ln \xi}{\partial \mathrm{T}}\right) d \mathrm{~T}+\left(\frac{\partial \ln \xi}{\partial \Pi}\right) d \Pi
$$

Fquating the last members of these two equations, we have

$$
\left(\frac{\partial \ln \xi^{\prime}}{\partial \mathrm{T}}\right) d \mathrm{~T}-\left(\frac{\partial \ln \xi}{\partial \mathrm{T}}\right) d \mathrm{~T}=\left(\frac{\partial \ln \xi}{\partial \Pi}\right) d \Pi \text {. }
$$

Now substituting for the partial differentials their values from the fundamental thermodynamic equations ${ }^{1}$ and combining the first two terms gives

$$
\frac{d \Pi}{d \mathrm{~T}}=\frac{-\mathrm{L}}{v \mathrm{~T}},
$$

where $\mathrm{L}$ is the heat absorbed in the fusion of one gram of ice and $v$ is the volume of one gram of water. For $T$ we may substitute $273.1-\Delta$, where $\Delta$ is the lowering of the freezing-point below the centigrade zero, whence

$$
\frac{d \Pi}{d \Delta}=\frac{L}{v(273 \cdot \mathrm{I}-\Delta)} .
$$

In order to integrate this equation, $L$ and $v$ must be known as functions of $\Delta$. According to a well-known principle the change of $I$ with $\Delta$ is given by the equation

$$
\mathrm{L}=\mathrm{L}_{0}-\mathrm{C} \Delta \text {, }
$$

where $\mathrm{L}_{0}$ is the heat of fusion at $0^{\circ} \mathrm{C}$. and $\mathrm{C}$ is the difference between the specific heats of water and ice. According to the best available data, $C$ is about 0.5 if our unit of energy is the calorie, or 21 if our unit of energy is the cc-atmos. The value of $L_{0}$ obtained in the very ac-

${ }^{1}$ Lewis: Loc. cit., equations V and VIII. It is of course to be noted that by definition $\Pi$ is a negative pressure. 
curate experiments of Smith ${ }^{1}$ was 334.2 joules, assuming the electromotive force of the Clark cell to be $\mathrm{I} .434 \mathrm{~V}$ at $15^{\circ}$. Since this value entered twice into the calculation of Smith, if we adopt for the Clark cell the value now accepted of $1.433 \mathrm{~V}$, the value of $\mathrm{I}_{00}$ must be lowered by 2 parts in 1434 and becomes 333.7 joules, or $3294 \mathrm{ce}$. -4tmos.

We may therefore write,

$$
\text { I. }=3294-21 \mathrm{~J} \text {. }
$$

Strictly speaking, $L$ is a function of the pressure also, but the pressure effect may easily be shown to be too small to be considered in the presint calculation.

The volume of a gram of water also depends upon both temperatur. and pressure. We shall see that one degree lowering of the freezing point corresponds to about is atmos. change in the osmotic pressure Hence from the known coefficients of thermal expansion and compressi bility we find that the value of $v$ may be expressed very closely by the linear equation,

$$
v=1.000+0.0008 \Delta \text {. }
$$

Substituting now in the above equation the values of $\mathrm{L}$ and $v$, and performing the multiplications and divisions indicated, we obtain $\frac{d l l}{d \Delta}$ its it series function of $\Delta$, namely,

$$
d \Delta=12.06-0.0414 \Delta-0.00009 \Delta^{2}+
$$

Except for very high values of $J_{\text {and }} J^{2}$ term and all the lighter ternis are negligible. Dropping these terms, therefore. and integrating. wh have

$$
\mathrm{Il}=\mathrm{I} 2.06 \Delta-0.2 \mathrm{I} \Delta^{2} .
$$

By this equation the osmotic pressure corresponding to any ircezing point lowering may be calctlated immediately and, if the experimentu! data used are as accurate as they appear, the error of the calculation can hardly exceed a few tenths of a per cent. even at osmotic pressures of several hundred atmospheres.

\section{Note 3 .}

The connection between the osmotic pressure and the vapor pressure of the solvent from a given solution is obtained as follows: From the fundamental thermodynamic equation for the change of the activity of a substance with the pressure, ${ }^{3}$ we have

'Phys. Rev., 17, 23I (1903).

$$
\frac{d l n}{d \mathrm{II}}=-\mathrm{V}
$$

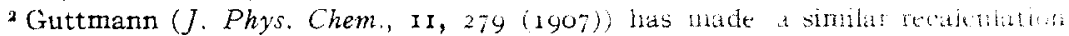
of Smith's value but applied only une-half of the correctinn applied above

- Lewis: Loc cit., equation $V$ 
where $\mathrm{V}$ is the molecular volume and $\xi$ is the activity of the pure solvent. When the vapor of the solvent behaves like a perfect gas whose pressure is $p$ we may write

Hence, in such a case,

$$
d \ln \xi=d \ln p
$$

$$
\frac{d \ln p}{d \Pi}=\frac{-\mathrm{V}}{\mathrm{RT}} .
$$

$\mathrm{V}$ may be regarded as constant for small values of II but at high pressures we must consider the compressibility of the solvent. If the coefficient of compressibility of the solvent is denoted by $\alpha$, and the volume when the osmotic pressure is zero by $V_{0}$,

$$
\mathrm{V}=\mathrm{V}_{0}(\mathrm{I}-\alpha \mathrm{II})
$$

Substituting this value of $\mathrm{V}$ in the above equation and integrating, we have

$$
\Pi-{ }_{2} \alpha \Pi^{2}=\frac{\mathrm{RT}}{\bar{V}_{0}} \ln \frac{p_{0}}{p}
$$

where $p_{0}$ is the vapor pressure of the pure solvent, $p$ that of the solvent in the solution of osmotic pressure $\Pi$. 'This equation is derived for the case that the vapor of the solvent obeys Boyle's law. In any other case a more complicated formula must be used.

\section{Summary.}

The simple laws of the infinitely dilute solution become mutually incompatible in solutions of finite concentration. It is therefore necessary to choose one law to serve as a criterion of the perfect solution. The only law of dilute solutions which ever holds in concentrated solutions is the law of Raoult. This law is stated in a slightly modified form and a perfect solution is defined as one which obeys this law. A number of solutions are mentioned which behave as perfect solutions over the whole range of concentrations, from o per cent. to 100 per cent. solute.

The indirect methods of determining osmotic pressure are discussed and an exact relation between the osmotic pressure and the freezingpoint lowering of an aqueous solution is obtained. It is also pointed out that the osmotic pressure at one temperature may be obtained from that at any other when the heat of dilution is known.

Adopting Raoult's law in its modified form as the characteristic law of the perfect solution, it is possible with the aid of thermodynamics alone to obtain an equation connecting the osmotic pressure and concentration of a perfect solution. The equation thus obtained permits the exact calculation of osmotic pressures in perfect solutions, up to I000 atmos. In comparatively dilute solutions the pressures thus obtained are substantially identical with those given by the equation of van't 
Hoff, as modified by Morse and Frazer, but at high concentrations the divergence between the two equations is very great.

An exact form is obtained for the mass law in concentrated perfect solutions.

Boston, March 2, 1905 .

[CONTRIBUTION FROM ROGER's LABORATORY OF PHYSTCS OF THE MASSACHUSETTS INSTITLTE OH TECHNOR.OOY]

\section{THE INDESTRUCTIBILITY OF MATTER AND THE ABSENCE OF EXACT RELATIONS AMONG THE ATOMIC WEIGHTS.}

H: DANELT. F. CONSTOCK.

keceived March 9 rops

The two chici reasons briefly stated for believing in the evolution of the elements one from another are, first, that some such process is un loubtedly taking place in the case of the radioactive substances, while we are being forced toward the conclusion that all the elements are radio active to some degree; and second, that in the hottest stars only two known elements occur, namely, hydroget and helium, while as we pass successively to cooler and cooler stars the other elements gradually make their appearance in a more or less orderly manner. Apparently this can only mean that at these transcendental temperatures the forces due to molecular or atomic impact are comparable with the interatomic forces involved in the breaking up of one element to form another, and hence the combination necessary for the formation of the heavier ele. ments can take place only after the temperature has sufficiently dropped.

There is one seemingly fatal objection, howerer, to any rery simple statement of the evolutionary theory and this objection has not been sufficiently emphasized. The diffeulty is this, that so far as we know there are no exact simple relations between the various atomic weights, whereas if we are to assume, as the simplest form of the evolutionary theory does, that the lighter elements come from an atomic disintegration of the heavier ones, or vice versa, it is evident that simple additive relations must exist.

As we know, many simple, additive relations do exist, but they are approximate, not exact, and the deviations from exactness, though sniall, are larger than we can explain from error in atomic weight determinations.

On the basis of common conceptions, therefore, the eridence seems contradictory, certain facts seeming to require the simple evolutionary idea, while another fact, the inexactness refered to above seming 6 deny it.

I wish to show that on the basis of the clectrical constitution at mat ter this inexactness is not only to be explained but it is to be expected 